Apidologie, 1978, 9 (4), 321-340.

\title{
OBSERVATIONS SUR LA POLLINISATION ENTOMOPHILE DE QUELQUES COUPLES D'HYBRIDES SIMPLES \\ DE CHOU FOURRAGER (Brassica oleracea $L$. var. acephala D.C.) EN ISOLEMENT POUR LA PRODUCTION DE SEMENCES D'HYBRIDES DOUBLES
}

\author{
Beobachtungen über die Insektenbestäubung einiger Paare \\ von Einfachhybriden des Blattkohls \\ (= Stauden-Winterkohl, Brassica oleracea L. var. acephala D.C.) \\ in isolierter Pflanzung zur Erzeugung von Doppelhybriden-Saatgut
}

\section{J. MESQUIDA}

I.N.R.A., Laboratoire de Recherches de la Chaire de Zoologie E.N.S.A., 65, rue de Saint-Brieuc, 35042 Rennes Cedex

\author{
SUMMARY \\ OBSERVATIONS ON ENTOMOPHILOUS POLLINATION OF A FEW PAIRS \\ OF SINGLE HYBRIDS OF FODDER KALE \\ (BRASSICA OLERACEA L. VAR. ACEPHALA D.C.) \\ IN ISOLATION FOR THE PRODUCTION OF DOUBLE HYBRID SEEDS
}

The cross-pollination of various kinds of single hybrids by local pollinators was studied in experimental plots used for creating double hybrids of fodder kales. Honeybees were the main pollinators and they played a prominent part in cross-pollination. Foraging was made at random without any precise orientation, without following the line or the row nor sticking to any line; plants had the same size and flowers the same colour. On some kinds of hybrid green cabbages there is an indirect foraging, which is perceptibly greater, and a difference of attractivity on behalf of red cabbages. Foraging is considered as indirect when honeybees alight outside the flower or at its corolla basis only to take nectar by parting the basis of petals. This position is unfavourable to pollination.

\section{RÉSUMÉ}

On a étudie la pollinisation croisée de divers types d'hybrides simples par les insectes pollinisateurs locaux dans des isolements servant à créer des hybrides doubles de choux fourragers. Les abeilles domestiques ont été les pollinisateurs essentiels et elles ont joué un rôle important dans la pollinisation croisée. Le 
butinage s'est effectué au hasard sans orientation précise, sans suivre la ligne ou le rang et sans fixation sur une lignée quelconque; les plantes étant de même taille et les fleurs de même couleur. On note seulement un butinage indirect sensiblement plus accentué sur certains types de choux verts hybrides et une différence d'attractivité en faveur des choux rouges. Précisons que le butinage est indirect lorsque les abeilles se posent extérieurement et à la base de la corolle de la fleur pour prélever uniquement le nectar en écartant légèrement la base des pétales. Cette position est défavorable à la pollinisation.

\section{INTRODUCTION}

Comme tous les autres choux, le chou fourrager est une plante à pollinisation entomophile mais qui présente des caractéristiques de l'auto-stérilité. C'est donc une plante à pollinisation croisée prépondérante, sinon obligatoire et qui, de plus, souffre beaucoup de la consanguinité par suite de la diminution de vigueur très marquée (effet d'inbreeding) qu'elle engendre. Selon du CreHu (1964), l'allogamie chez le chou fourrager est due au phénomène d'auto-incompatibilité pollinique qu'il a mis en évidence mais qui était déjà signalé depuis longtemps sur d'autres types de choux (Brassica oleracea L.) par Roemer (1916); Pearson (1929) et Attia et Munger (1950). Depuis lors, le phénomène d'auto-incompatibilité pollinique chez le chou a fait l'objet de nombreux travaux qui ont permis d'apporter d'importantes précisions tant sur sa physiologie que sur son déterminisme génétique. Les conclusions essentielles de ces travaux montrent bien que l'incompatibilité entre pollen et style se traduit par l'inaptitude du pollen à féconder l'ovule de la même plante ou d'une plante génétiquement identique. Plus précisément dans le cas du chou, comme chez tous les Brassica concernés le phénomène se caractérise par l'inhibition de la germination du pollen sur le stigmate et il est de nature biochimique. Il n'apparait que tardivement au cours du développement de la fleur, atteint son maximum à l'anthèse et peut alors être plus ou moins intense suivant les individus c'est-à-dire suivant le génotype de la plante. L'allogamie n'est généralement pas absolue chez le chou, mais dans les conditions normales de la pollinisation elle peut être quasi totale grâce à la compétition pollinique favorisant le pollen des plantes génétiquement différentes (allo-pollen) par rapport à celui de la plante (autopollen). Pour obtenir un taux maximum de semences hybrides et un bon rendement en graines, il importe donc que les fleurs de chou fourrager reçoivent en abondance du pollen de plantes génétiquement différentes. Ce sont les insectes qui effectuent généralement cette pollinisation et la présence de siliques développées avec graines est un critère qui permet de reconnaître facilement si la plante a été pollinisée ou non.

Ainsi ce mode de fécondation du chou fourrager joue un rôle important en matière de sélection, en particulier pour la création de variétés hybrides susceptibles d'être plus productives que les variétés traditionnelles.

Les travaux d'amélioration du chou fourrager poursuivis à la Station d'Amélioration des plantes de Rennes (I.N.R.A.) portent essentiellement sur l'exploitation de ces phénomènes d'auto-incompatibilité pollinique et ont pour objectif la création de variétés hybrides doubles. Les essais de production d'hybrides sont conduits dans des isole- 
ments de surfaces très restreintes mis en place en plein champ. C'est dans de tels isolements que nous avons effectué en 1976 des observations sur la pollinisation en conditions naturelles. Il s'agissait de faire un inventaire de la faune pollinisatrice, d'étudier le comportement de butinage de l'Abeille domestique et de vérifier l'effet de l'action pollinisatrice.

\section{MATÉRIEL ET MÉTHODE}

\section{1. - Matériel végétal}

Le matériel végétal était constitué de 4 isolements (tabl. 1) de 8 lignes et 5 plantes par ligne soit 40 plantes dont 20 d'un hybride simple de chou Demi-Moellier vert et 20 d'un hybride simple de chou Cavalier rouge. Les choux verts et rouges étaient disposés soit en lignes alternées soit en plantes alternées avec un espacement de $0,70 \mathrm{~m}$.

TABL. 1. - Répartition des divers couples d'hybrides de chou fourrager dans les isolements.

TAB. 1. - Verteilung der verschiedenen Paare von Einfachhybriden des Blattkohls in den isolierten Feldern.

\begin{tabular}{c|l|c|c|c}
\hline \hline \multirow{2}{*}{$\begin{array}{c}\text { No de l'isolement } \\
\text { Nr. des isolierten Feldes }\end{array}$} & $\begin{array}{c}\text { Nom du lieu } \\
\text { Standort }\end{array}$ & \multicolumn{2}{|c|}{$\begin{array}{c}\text { No des hybrides } \\
\text { Nr. der Hybriden }\end{array}$} & \multirow{2}{*}{$\begin{array}{c}\text { Plantation } \\
\text { Art der Pflanzung }\end{array}$} \\
\cline { 3 - 5 } & & $\mathrm{V}$ & $\mathrm{R}$ & \\
\hline 1 & La Motte & $(429 \times 813)$ & $(919 \times 155)$ & PA \\
2 & Méjussaume & $\begin{array}{l}(429 \times 813) \\
(429 \times 905) \\
(429 \times 905)\end{array}$ & $\begin{array}{l}(111 \times 155) \\
(111 \times 155)\end{array}$ & LA \\
4 & Grand Noël & Haute Division & $(155)$ & LA \\
\hline \hline
\end{tabular}

$\mathrm{V}=$ choux verts

PA $=$ plantes alternees

$\mathrm{V}=$ Grüner Blattkohl

$\mathrm{PA}=$ alternierende Pflanzen

$$
\begin{aligned}
& \mathrm{R}=\text { choux rouges } \\
& \mathrm{LA}=\text { lignes alternees } \\
& \mathrm{R}=\text { Roter Blattkohl } \\
& \mathrm{LA}=\text { alternierende Reihe }
\end{aligned}
$$

\section{2. - Observations effectuées}

- L'évolution des floraisons a été estimée périodiquement en effectuant des comptages de fleurs butinables par la méthode du cerceau de $50 \mathrm{~cm}$ de diamètre préconisée par DelaUdE et TASEI (1972).

- L'inventaire des insectes pollinisateurs a été effectué à différentes dates et à différents moments de la journée par comptages au passage dans les rangs de chaque isolement.

- Les abeilles domestiques visitant directement et indirectement les fleurs de chou fourrager ont été notées à différentes reprises. Les visites sont dites directes lorsque les abeilles se posent normalement sur la fleur ouverte pour butiner soit du nectar, soit du pollen ou les deux à la fois selon les cas : la pollinisation est alors possible. Elles sont dites indirectes lorsque les abeilies se posent extérieurement et à la base de la corolle, bien que la fleur soit normalement ouverte, pour prélever uniquement du nectar. Dans cette posi- 
tion, qui est indiscutablement défavorable à la pollinisation, les abeilles accèdent facilement aux nectaires en écartant légèrement la base des pétales (fig. 1).

L'étude préférentielle du butinage a été effectuée par comparaisons des densités d'abeilles domestiques pour 1000 fleurs butinables du chou rouge et du chou vert.

La vitesse de butinage a été calculée en nombre de fleurs visitées à la minute. Le nombre de fleurs visitées par pied a été également noté de même que les fréquences de passages des abeilles d'un pied à un autre pour l'étude du butinage alterné.

Le trajet effectué par les abeilles domestiques et les bourdons pour butiner les fleurs de chou fourrager en isolement a été suivi et représenté graphiquement. Le nombre de fleurs butinées par pied a été, au cours de cette observation, soigneusement noté.

L'activité journalière de butinage des insectes pollinisateurs sur fleurs de chou fourrager a été observée en 1966 pendant la période du maximum de floraisons et au cours d'une journée complète entre $7 \mathrm{~h}$ et $18 \mathrm{~h}$ (heures solaires) et par beau temps. Les comptages ont été effectués toutes les 15 minutes sur de petites parcelles bien délimitées $\left(8 \mathrm{~m}^{2}\right.$ en tout) d'un champ d'une centaine de mètres carrés.

L'efficacité pollinisatrice a été vérifiée en calculant le taux de nouaison :

$$
\left(\frac{\text { nombre de siliques formées }}{\text { nombre total de fleurs }} \times 100\right)
$$

des 20 premières fleurs de 5 rameaux par pied. Ces prélevements concernent 5 pieds de choux verts et de choux rouges par isolement; les rameaux et les pieds étant tirés au hasard. Le nombre de graines par silique

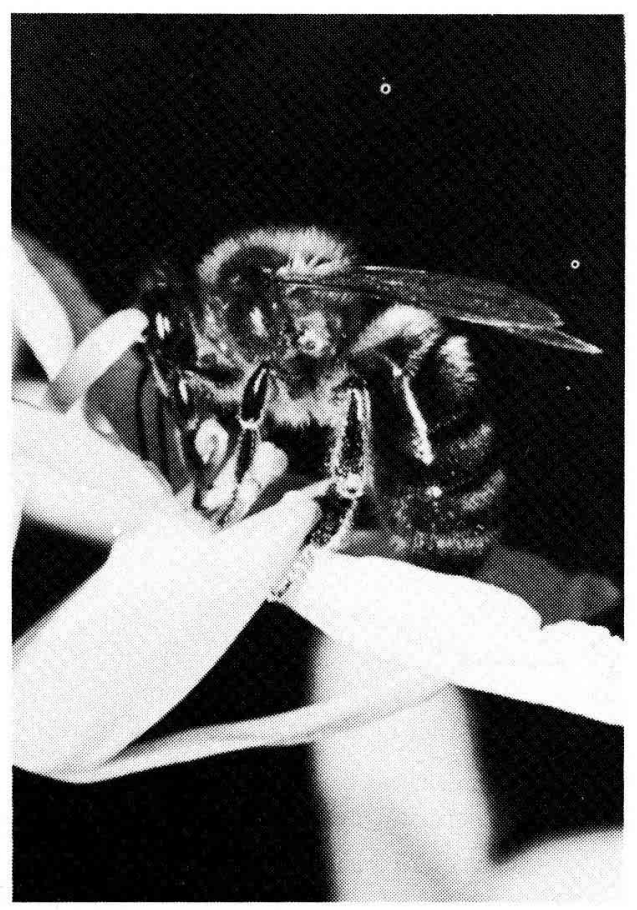

FIG. 1 a. - Visite directe des fleurs de chou fourrager par l'abeille domestique (photo CARRE).

ABв. 1 a. - Direkter Blütenbesuch beim Blattkohl durch die Honigbiene (photo CARRE). 

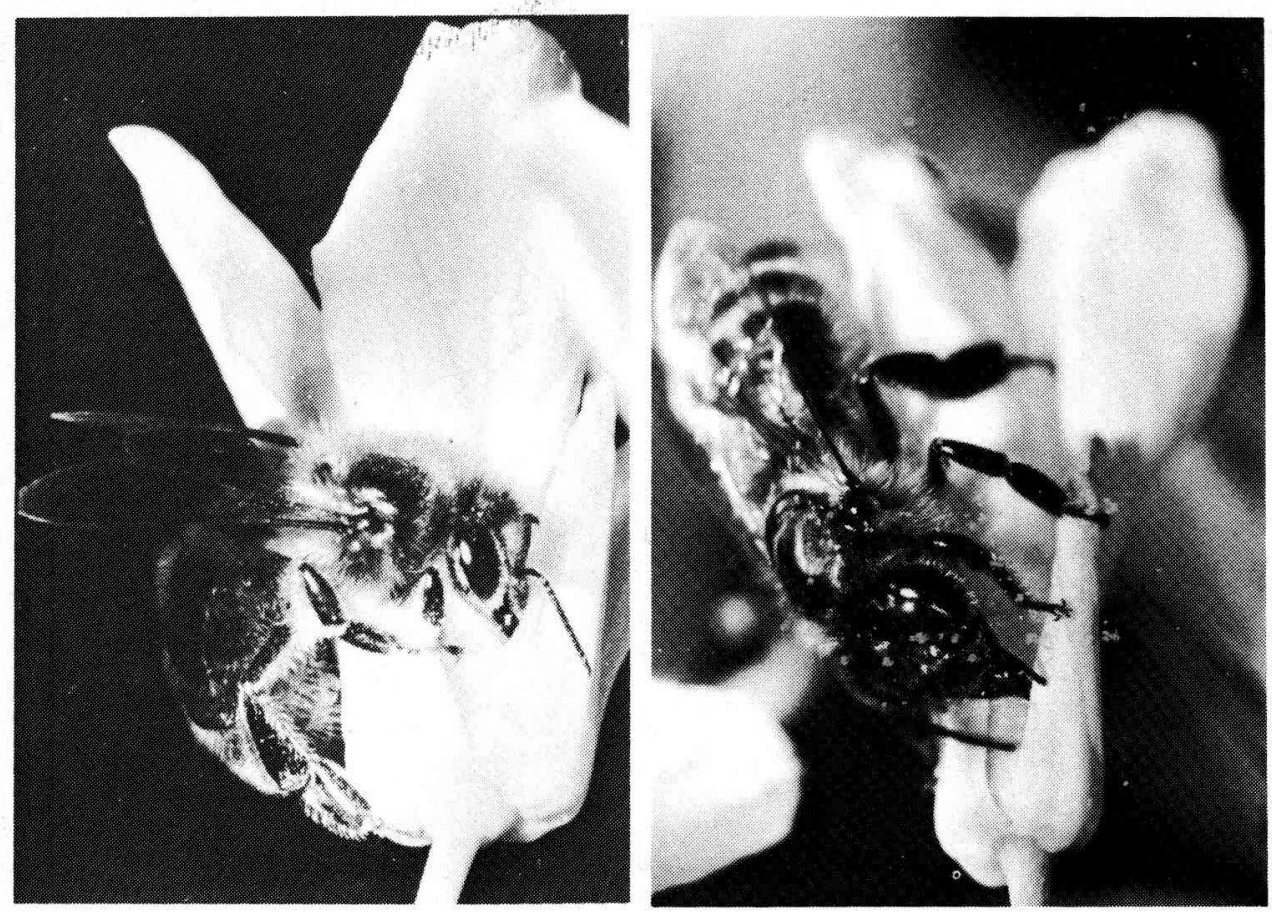

FIG. $1 \mathrm{~b}$ et c. - Visites indirectes des fleurs de chou fourrager par l'abeille domestique (photo CARRE).

ABB. 1 b und c. - Indirekte Blütenbesuche beim Blattkohl durch die Honigbiene (photo CARRE).

et le poids de 1000 graines ont été également comptés sur ces prélèvements. Les poids de graines pour 1000 fleurs butinables ont été calculés en fonction du taux de nouaison, du nombre de graines par silique et du poids de 1000 graines obtenus.

\section{RÉSULTATS}

\section{1. - Évolution des floraisons}

Les floraisons des choux verts et des choux rouges de tous les isolements se sont produites en même temps (fig. 2). Elles ont duré près de six semaines. Elles ont toutes débuté vers le 5 avril et elles se sunt terminées ensemble vers le 17 mai. C'est vers la fin du mois d'avril que l'on a noté le maximum de floraison. On s'aperçoit cependant que les floraisons ont été plus abondantes tantôt chez les choux verts dans les isolements $\mathrm{n}^{\circ} 1$ et $\mathrm{n}^{\circ} 4$ et tantôt chez les choux rouges dans les isoiements $\mathrm{n}^{\circ} 2$ et $\mathrm{n}^{\mathrm{o}} 3$. 

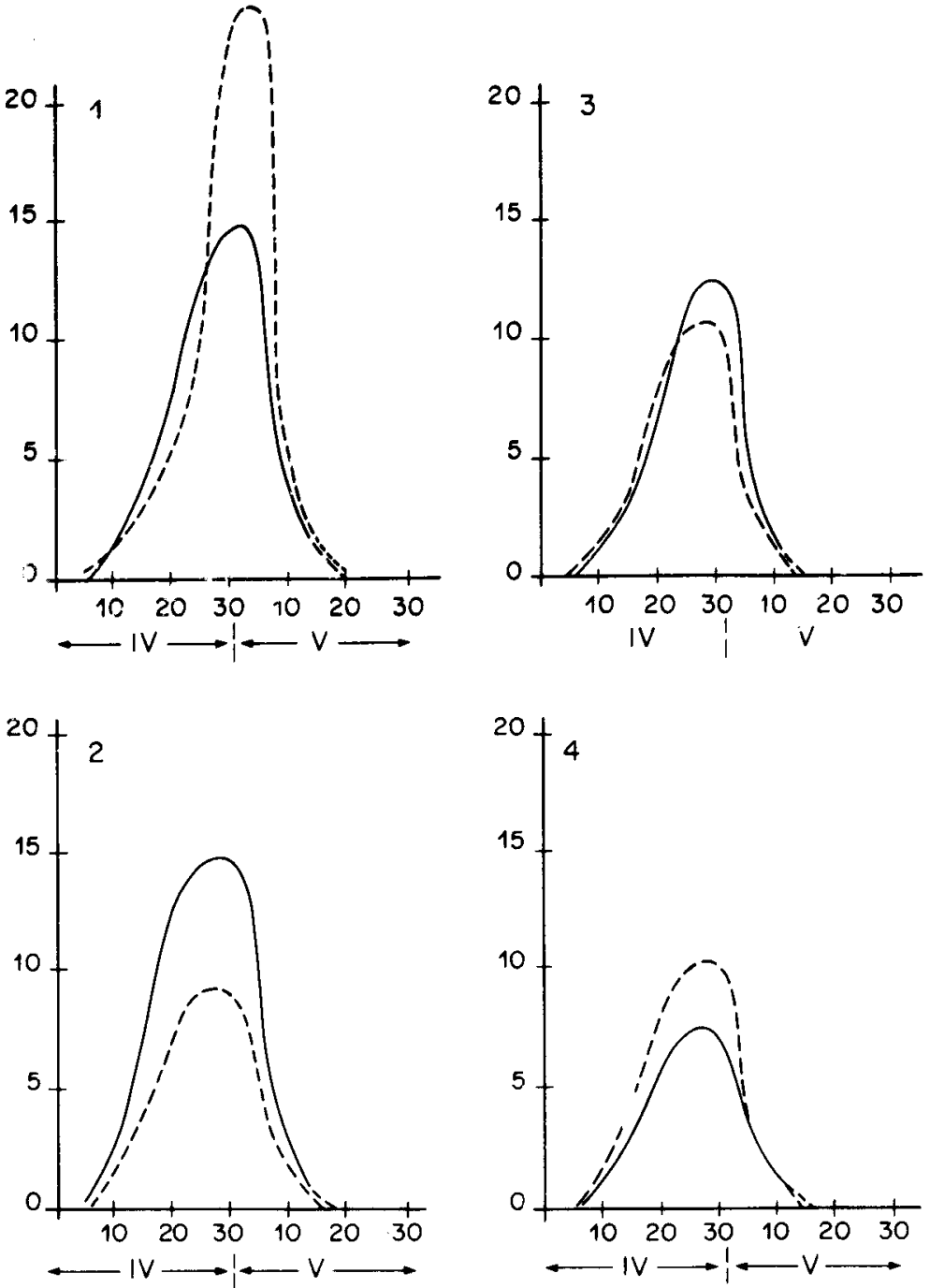

FIG. 2. - Evvolution des floraisons de choux fourragers à Rennes en 1976.

en abscisse : mois avril (IV) et mai (V)

en ordonnée : nombre de fleurs au $\mathrm{m}^{2}(n \times 100)$

1 pour isolement $n^{\circ} 1 ; 2$ pour isolement $n^{\circ} 2$;

3 pour isolement $n^{\circ} 3 ; 4$ pour isolement $n^{\circ} 4$

AвB. 2. - Verlauf der Blüte des Blattkohls in Rennes im Jahre 1976.

Abszisse : Monate April (IV) und Mai (V)

Ordonate : Zahl der Blüten je $\mathrm{m}^{2}(n \times 100)$

I $=$ isoliertes Feld Nr. 1; $2=$ isoliertes Feld Nr. 2;

$3=$ isoliertes Feld Nr. 3; $4=$ isoliertes Feld Nr. 4 


\section{2. - Faune pollinisatrice}

L'inventaire des insectes pollinisateurs visitant les choux fourragers, effectué du 8 au 30 avril 1976 (tabl. 2) permet de montrer que ce sont surtout les abeilles domestiques (Apis m. mellifica L.) qui ont dominé très largement. Elles ont constitué $86,3 \%$ de la population globale. Les bourdons n'ont constitué que $5,4 \%$ de la population globale et n'ont pas été plus nombreux que les abeilles sauvages diverses $(7,2 \%)$ : (Anthophore : $3,1 \%$; Osmies : $1,3 \%$; Andrènes : $0,6 \%$; Eucera : $0,2 \%$ et autres abeilles sauvages : $2 \%$ ). Les Diptères $(0,7 \%)$ et les Lépidoptères $(0,4 \%)$ ont été très rares.

Les bourdons ont été représentés surtout par les Bombus terrestris $L$. et les Bombus lapidarius $\mathrm{L}$. Les autres espèces : $B$. hortorum, $B$. agrorum et $B$. pratorum ont été peu nombreuses.

TABL. 2. - Insectes pollinisateurs des choux fourragers hybrides simples en isolement pendant la période du 8 au 30 avril à Rennes.

TAB. 2. - Insekten als Bestäuber von Einfachhybriden des Blattkohls auf isolierten Feldern in der Zeit vom 8.-30. April 1976 in Rennes.

\begin{tabular}{|c|c|}
\hline $\begin{array}{l}\text { Pollinisateurs } \\
\text { Bestäuber }\end{array}$ & $\begin{array}{l}\text { Effectif (en \%) } \\
\text { Anteil (in \%) }\end{array}$ \\
\hline $\begin{array}{l}\text { Abeilles domestiques } \ldots \ldots \ldots \ldots \ldots \ldots \ldots \ldots \ldots \ldots \ldots \ldots \\
\text { Honigbienen }\end{array}$ & 86,3 \\
\hline $\begin{array}{l}\text { Bourdons } \ldots \ldots \ldots \ldots \ldots \ldots \ldots \ldots \ldots \ldots \ldots \ldots \ldots \ldots \ldots \\
\text { Hummeln }\end{array}$ & 5,4 \\
\hline $\begin{array}{l}\text { Anthophore } \\
\text { Anthophora }\end{array}$ & 3,1 \\
\hline $\begin{array}{l}\text { Osmies } \\
\text { Osmia }\end{array}$ & 1,3 \\
\hline $\begin{array}{l}\text { Andrènes } \ldots \ldots \ldots \ldots \ldots \ldots \\
\text { Andrena }\end{array}$ & 0,6 \\
\hline $\begin{array}{l}\text { Eucera } \\
\text { Eucera }\end{array}$ & 0,2 \\
\hline $\begin{array}{l}\text { Autres Abeilles sauvages } \ldots \ldots \ldots \ldots \ldots \ldots \ldots \ldots \ldots \ldots \ldots \\
\text { Andere Wildbienen }\end{array}$ & 2 \\
\hline $\begin{array}{l}\text { Diptères } \\
\text { Dipteren }\end{array}$ & 0,7 \\
\hline $\begin{array}{l}\text { Lépidoptères } \ldots \ldots \ldots \ldots \ldots \ldots \ldots \ldots \ldots \ldots \ldots \ldots \ldots \ldots \\
\text { Lepidopteren }\end{array}$ & 0,4 \\
\hline
\end{tabular}




\section{3. - Comportement de butinage}

a) Particularité du comportement de récolte de l'Abeille domestique

Les visites des fleurs de chou fourrager par l'Abeille domestique ont eu tendance à s'effectuer de manières différentes. Elles ont été plus souvent directes mais le tableau 3 montre que l'on a observé aussi des visites indirectes plus ou moins importantes selon les hybrides simples. D'ailleurs, en fonction de l'importance du pourcentage de butinage indirect observé il est possible de faire ressortir des groupes différents:

TABL. 3. - Variations du butinage direct ou indirect sur les floraisons de divers hybrides simples de choux fourragers.

ТАВ. 3. - Variation direkter oder indirekter Sammeltätigkeit auf den Blüten verschiedener Einfachhybriden des Blattkohls.

\begin{tabular}{|c|c|c|c|c|c|}
\hline \multirow{2}{*}{$\begin{array}{c}N^{0} \text { de l'isolement } \\
\text { Nr. des isolierten Feldes }\end{array}$} & \multirow{2}{*}{$\begin{array}{l}N^{\circ} \text { des hybrides simples } \\
\text { Nr. der Einfachhybriden }\end{array}$} & \multicolumn{2}{|c|}{$\begin{array}{l}\text { Butinage direct } \\
\text { Direktes Sammeln }\end{array}$} & \multicolumn{2}{|c|}{$\begin{array}{l}\text { Butinage indirect } \\
\text { Indirektes Sammeln }\end{array}$} \\
\hline & & & $(\%)$ & $\mathrm{n}$ & $(\%)$ \\
\hline 1 & $\begin{array}{l}V(429 \times 813) \\
R(919 \times 155)\end{array}$ & $\begin{array}{r}3 \\
43\end{array}$ & $\begin{array}{l}(15,8) \\
(98,9)\end{array}$ & $\begin{array}{r}16 \\
1\end{array}$ & $\begin{array}{r}(84,2) \\
(1,1)\end{array}$ \\
\hline 2 & $\begin{array}{l}\mathrm{V}(429 \times 813) \\
\mathrm{R}(111 \times 155)\end{array}$ & $\begin{array}{r}7 \\
46\end{array}$ & $\begin{array}{l}(22,6) \\
(77,1)\end{array}$ & $\begin{array}{l}24 \\
14\end{array}$ & $\begin{array}{l}(77,4) \\
(22,9)\end{array}$ \\
\hline 3 & $\begin{array}{l}\mathrm{V}(429 \times 905) \\
\mathrm{R}(919 \times 155)\end{array}$ & $\begin{array}{r}7 \\
49\end{array}$ & $\begin{array}{l}(100) \\
(100)\end{array}$ & $\begin{array}{l}0 \\
0\end{array}$ & $\begin{array}{l}(0) \\
(0)\end{array}$ \\
\hline 4 & $\begin{array}{l}V(429 \times 905) \\
R(111 \times 155)\end{array}$ & $\begin{array}{l}13 \\
52\end{array}$ & $\begin{array}{l}(96,3) \\
(98,1)\end{array}$ & $\begin{array}{l}1 \\
1\end{array}$ & $\begin{array}{l}(3,7) \\
(1,9)\end{array}$ \\
\hline
\end{tabular}

$\mathrm{V}=$ choux fourragers verts.

$\mathrm{R}=$ choux fourragers rouges.

$\mathrm{V}=$ Grüner Kohl.

R = Roter Kohl.

- le premier dont les visites indirectes ont été très fréquentes $(\geqslant 70 \%)$ : choux verts $(429 \times 813)$ des isolements $n^{0} 1$ et 2 (tabl. 1 et 3 );

- le deuxième dont les visites indirectes ont été assez fréquentes $(\geqslant 20 \%$ et $<70 \%)$ : choux rouges $(111 \times 155)$ de l'isolement $n^{\circ} 2$ (tabl. 1 et 3$)$;

- enfin le troisième dont les visites ont été nulles ou faibles: choux verts $(429 \times 905)$ et choux rouges $(919 \times 155)$ de l'isolement $n^{\circ} 3$; choux verts $(429 \times 905)$ et choux rouges $(111 \times 155)$ de l'isolement $n^{0} 4$; choux rouges $(919 \times 155)$ de l'isolement $n^{\circ} 1$ (tabl. 1 et 3 ).

Ainsi, chez les choux verts le butinage indirect est du premier groupe quand on est en présence de la combinaison hybride $(429 \times 813)$; il est par contre du troisième groupe avec la combinaison $(429 \times 905)$. 
Chez les choux rouges le butinage indirect est généralement du $3^{e}$ groupe mais avec la combinaison hybride $(111 \times 155)$ il peut être aussi du second groupe.

Le butinage indirect semble donc s'être produit plus fréquemment chez les choux verts de la combinaison hybride $(429 \times 813)$ que chez ceux de la combinaison $(429 \times 905)$. Les choux rouges ont été intermédiaires.

Notons aussi que les mêmes abeilles ont été susceptibles de butiner soit directement, soit indirectement, de même que des fleurs visitées indirectement par une abeille ont été aussi visitées directement par d'autres abeilles.

b) Préférence de butinage de l'Abeille domestique

La densité moyenne d'abeilles pour 1000 fleurs butinables a été toujours plus élevée sur les fleurs de choux rouges de chaque isolement que celle qui a été observée sur fleurs de choux verts (tabl. 4). Les abeilles ont visité en moyenne 2,2 fois plus de fleurs de choux rouges que de fleurs de choux verts. Statistiquement ces différences sont significatives. L'Abeille domestique semble donc manifester une préférence de butinage sur fleurs de choux rouges.

TABL. 4. - Densités moyennes d'abeilles domestiques pour 1000 fleurs butinables de divers hybrides simples de choux fourragers.

Tав. 4. - Mittlere Bienendichte auf 1000 geöffnete Blüten von verschiedenen Einfachhybriden des Blattkohls.

\begin{tabular}{|c|c|c|c|c|c|c|}
\hline \multirow[b]{2}{*}{$\begin{array}{c}\mathrm{N}^{0} \text { de l'isolement } \\
\mathrm{Nr} \text {. des isolierten Feldes }\end{array}$} & \multicolumn{3}{|c|}{$\mathrm{V}$} & \multicolumn{3}{|c|}{$\mathrm{R}$} \\
\hline & $\begin{array}{l}\mathrm{N}^{0} \text { des hybrides } \\
\text { simples } \\
\text { Nr. der Einfach- } \\
\text { hybriden }\end{array}$ & D & $(\%)$ & $\begin{array}{l}\mathrm{N}^{0} \text { des hybrides } \\
\text { simples } \\
\text { Nr. der Einfach- } \\
\text { hybriden }\end{array}$ & $\mathrm{D}$ & (\%) \\
\hline $\begin{array}{l}1 \\
2 \\
3 \\
4 \\
\end{array}$ & $\begin{array}{l}(429 \times 813) \\
(429 \times 813) \\
(429 \times 905) \\
(429 \times 905) \\
\end{array}$ & $\begin{array}{l}0,79 \\
1,61 \\
0,03 \\
2,07 \\
\end{array}$ & $\begin{array}{l}(32,2) \\
(41,9) \\
(1,5) \\
(34,6) \\
\end{array}$ & $\begin{array}{l}(919 \times 155) \\
(111 \times 155) \\
(919 \times 155) \\
(111 \times 155) \\
\end{array}$ & $\begin{array}{l}1,66 \\
2,23 \\
1,94 \\
3,92 \\
\end{array}$ & $\begin{array}{l}(67,7) \\
(58) \\
(98,5) \\
(65,4) \\
\end{array}$ \\
\hline $\begin{array}{l}\bar{m} \\
s / \sqrt{n} \\
\%\end{array}$ & & $\begin{array}{l}1,13 \\
0,45 \\
(1\end{array}$ & $\begin{array}{l}(31,7) \\
\%)\end{array}$ & & $\begin{array}{l}2,44 \\
0,51 \\
\quad(2\end{array}$ & $\begin{array}{l}(68,3) \\
\%)\end{array}$ \\
\hline $\begin{array}{l}\text { Différences } \\
\text { Unterschied }\end{array}$ & & & & $*(\mathbf{P}=0,05)$ & & \\
\hline
\end{tabular}

$\mathrm{V}==$ Choux fourragers verts.

$\mathrm{R}=$ Choux fourragers rouges.

$\mathrm{D}=$ Densité moyenne d'abeilles pour 1000 fleurs butinables.

$\mathrm{V}=$ Grüner Kohl.

$\mathrm{R}=$ Roter Kohl.

$\mathrm{D}=$ Mittlere Bienendichte pro 1000 geöffnete Blüten. 


\section{c) Vitesses de butinage}

Dans l'ensemble, les abeilles domestiques ont visité en moyenne 5,85 $( \pm 0,56)$ fleurs à la minute. Ces visites ont été cependant plus rapides sur fleurs de choux rouges $(\overline{\mathrm{m}}=9 \pm 0,4)$ que sur fleurs de choux verts $(\overline{\mathrm{m}}=4,24 \pm 0,92)$. Notons que les abeilles ont eu aussi une légère tendance à visiter un peu plus de fleurs par pied sur choux rouges $(\overline{\mathrm{m}}=5,71 \pm 1,08)$ que sur choux verts $(\overline{\mathrm{m}}=4,75 \pm 0,67)$. Le nombre de fleurs visitées par pied ne semble pas en tous cas dépendre du nombre de fleurs ouvertes et butinables. Les corrélations obtenues n'ont jamais été significatives.

Les bourdons ont été beaucoup plus rapides que les abeilles de même qu'ils ont visité plus de fleurs par pied. Ils ont visité en moyenne $13,88( \pm 2,03)$ fleurs à la minute. Ils ont visité en moyenne par pied $9,07( \pm 4,78)$ fleurs de choux rouges et $6,93( \pm 1,43)$ fleurs de choux verts.

\section{d) Butinage alterné de l'A beille domestique}

Sur 17 abeilles observées du 7 au 27 avril ayant visité en tout et successivement des fleurs de 82 pieds différents, la fréquence de l'alternance entre pieds différents et entre pieds identiques a été la suivante :

- 44 passages entre pieds identiques (ou $53 \%$ );

- et 38 passages entre pieds différents (ou $47 \%$ ).

Ces résultats montrent que l'alternance du butinage entre choux verts et choux rouges semble avoir été ainsi assurée.

e) Trajet des abeilles domestiques et des bourdons pour le butinage des choux fourragers en isolement

Pour butiner les fleurs de choux verts et de choux rouges appartenant à des plantes de taille identique, en plantation alternée ou en ligne alternée, le trajet effectué par l'Abeille domestique et les bourdons (Bombus terrestris; $B$. lapidarius; $B$. hortorum; $B$. agrorum) dans les isolements semble s'effectuer uniquement au hasard. En effet, les figures 3 et 4 font apparaître des contours d'un cheminement très irrégulier sans aucune orientation bien précise. Les insectes ne suivent ni la ligne ni le rang d'un bout à l'autre de l'isolement. Les figures 3 et 4 permettent aussi de remarquer que s'il y a eu parfois tendance, aussi bien chez l'A beille que chez le Bourdon, à suivre une même ligne pendant un court instant il y a eu néanmoins changement d'orientation assez rapidement. Les passages entre pieds différents situés immédiatement à côté les uns des autres se sont souvent produits au cours du cheminement mais les passages successifs sur des pieds identiques ou différents et très éloignés les uns des autres se sont également produits. 


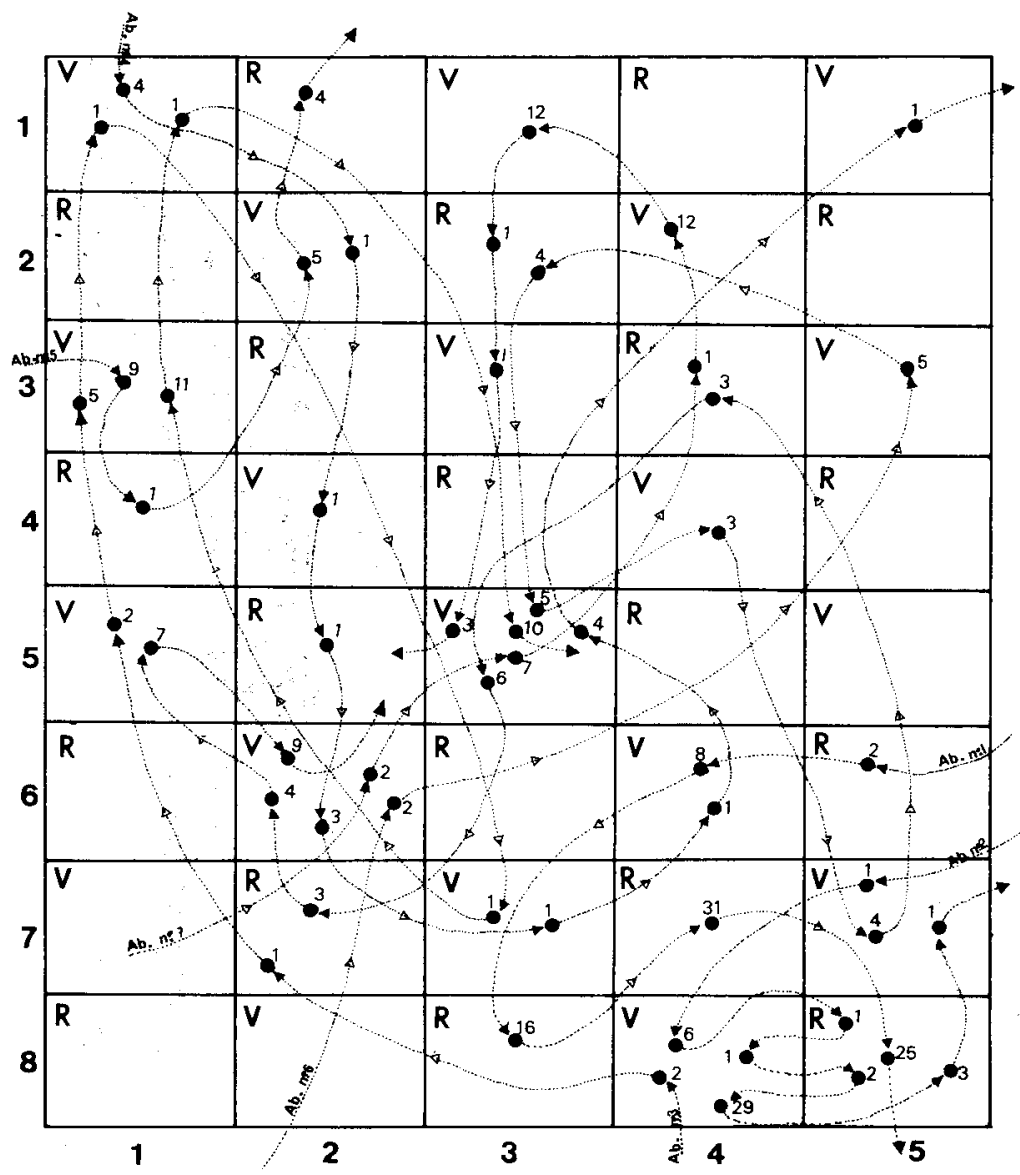

FIG. 3. - Schéma du cheminement de 7 abeilles domestiques

dans un isolement de choux rouges et choux verts en plantation alternée.

en abscisse : les lignes

en ordonnée : les rangs

chaque rectangle correspond à un pied de chou

$\rightarrow \quad$ sens du cheminement

- 5 butinage sur 5 fleurs du même pied avant de passer au pied suivant

$\mathrm{V}=$ chou vert

$\mathbf{R}=$ chou rouge

$\mathrm{Ab}=$ Abeille

Авв. 3. - Schema der Flugbahnen von 7 Honigbienen in einem isolierten Feld von rotem und grünem Blatkohl bei alternierender Pflanzung.

Abszisse : Die Linien

Ordinate : Die Reihen

Jedes Rechteck entspricht einer Kohlpflanze

$\rightarrow \quad$ Richtung der Flugbahn

- 5 Sammeln an 5 Blüten derselben Pfianze vor dem Weiterflug zur nächsten Pflanze.

V Grüner Blattkohl

R Roter Blattkohl

Ab Biene 


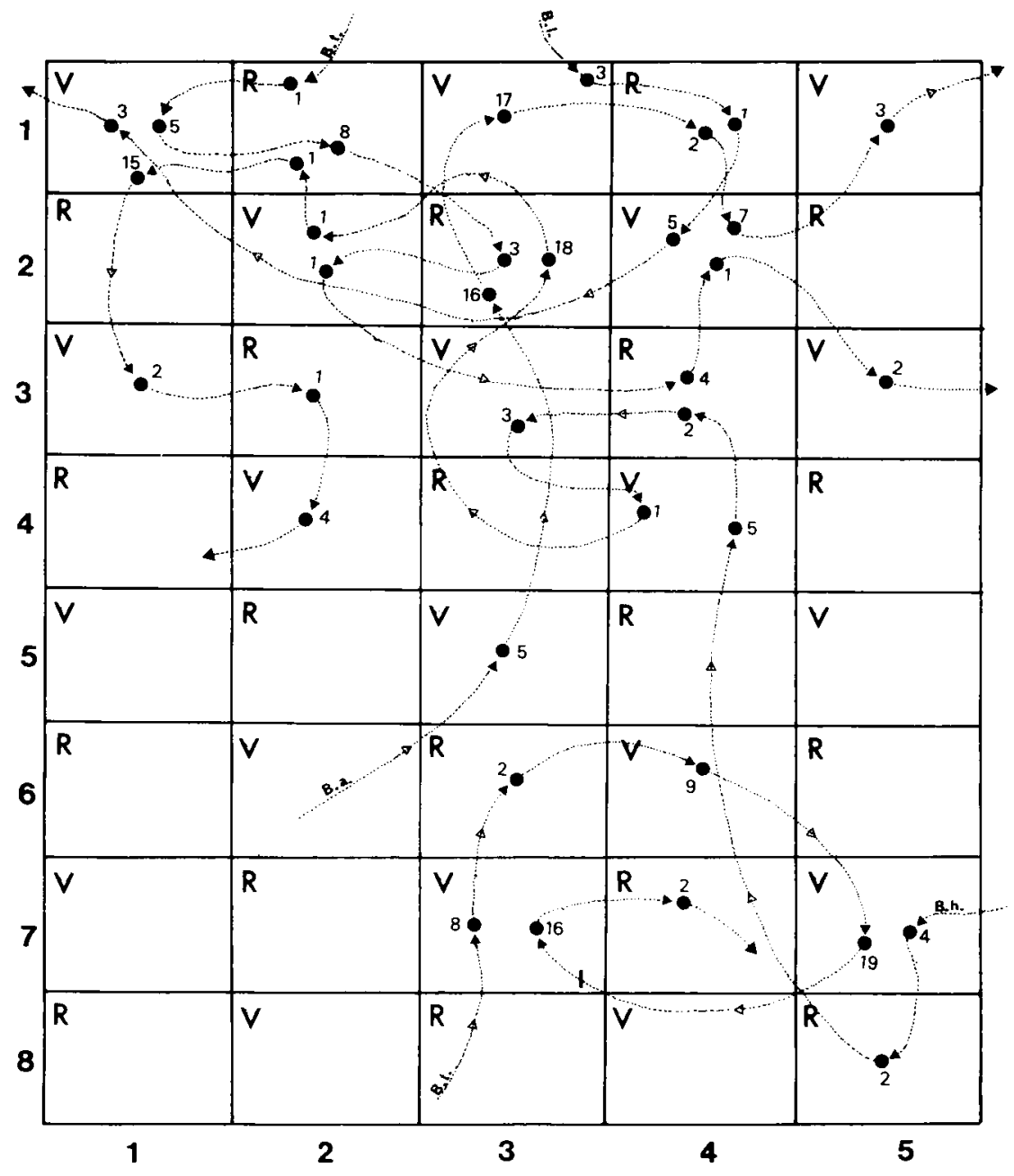

FIG. 4. - Schéma du cheminement de 5 fondatrices de bourdons dans un isolement de choux rouges et de choux verts en plantation alternée.

Même légende que fig. 3

$$
\begin{aligned}
& \mathrm{Bt}=\text { Bombus terrestris } \\
& \mathrm{B} \mathrm{l}=\text { Bombus lapidarius } \\
& \mathrm{Bh}=\text { Bombus hortorum } \\
& \mathrm{Ba}=\text { Bombus agrorum }
\end{aligned}
$$

Авв. 4. - Schema der Flugbahnen von 5 Hummelweibchen in einem isolierten Feld

mit rotem und grünem Blatlkohl bei alternierender Pflanzung.

Dieselbe Legende wie in Abb. 3

$$
\begin{aligned}
& \mathrm{Bt}=\text { Bombus terrestris } \\
& \mathrm{Bl}=\text { Bombus lapidarius } \\
& \mathrm{Bh}=\text { Bombus hortorum } \\
& \mathrm{Ba}=\text { Bombus agrorum }
\end{aligned}
$$




\section{f) Activité journalière du butinage}

Sur un total de 927 pollinisateurs recensés l'activité journalière de butinage observée s'est effectuée de la façon suivante :

Les abeilles domestiques ont commencé à butiner à 8 heures et se sont arrêtées à $16 \mathrm{~h} 45$. Elles ont éte plus nombreuses le matin entre 9 heures et 12 heures $(55,5 \%)$ que l'après-midi entre 12 heures et 18 heures (44,5\%). Le maximum de butinage s'est situé entre 9 heures et 11 heures $(46,4 \%)$. On a noté une petite reprise d'activité tardive entre 16 heures et 17 heures.

Les bourdons ont commencé à butiner à peu près à la même heure que les abeilles ( $7 \mathrm{~h} 45$ ) mais ils ont terminé plus tard ( 18 heures). Contrairement aux abeilles ils ont été plus fréquents l'après-midi (64\%) que le matin (36\%). Ils ont été environ deux fois plus nombreux l'après-midi.

Les autres abeilles sauvages ont butiné de $9 \mathrm{~h} 30$ à 15 heures. Elles ont été six fois plus nombreuses le matin ( $86 \%$ ) que l'après-midi (14\%).

\section{4. - Efficacité pollinisatrice}

\section{a) Taux de nouaison}

Les nouaisons ont été satisfaisantes dans l'ensemble (tabl. 5), sauf chez les choux verts et les choux rouges de l'isolement $n^{\circ} 2$ chez qui les visites indirectes par les abeilles domestiques ont été très fréquentes à assez fréquentes (tabl. 3). L'analyse statistique fait d'ailleurs ressortir des différences significatives ou hautement significatives. Mais les choux verts de l'isolement $n^{\circ} 1$ dont le butinage indirect a été également très fréquent a cependant donné une nouaison significativement meilleure que celle des choux verts identiques de l'isolement $n^{\circ} 2$. Il est remarquable de constater que les choux rouges, plus attractifs, ont eu tendance à fournir, à l'exception de l'isolement $n^{\circ} 3$, des taux de nouaison sensiblement plus élevés que les choux verts.

b) Nombre de graines par silique, poids de 1000 graines et poids de graines pour 1000 fleurs butinables

Les moyennes du nombre de graines par silique, du poids des graines et des rendements en graines ont été groupées dans le tableau 5. Là aussi l'analyse statistique a permis de faire apparaitre des différences significatives entre les moyennes du nombre de graines par silique et du poids de 1000 graines chez les choux rouges seulement. $\mathrm{Ce}$ sont les choux rouges de l'isolement $n^{\circ} 2$ qui ont eu des moyennes significativement plus élevées, d'une part en ce qui concerne le nombre de graines par silique et plus faibles, d'autre part en ce qui concerne le poids des graines. Le poids des graines pour 1000 fleurs de choux rouges de l'isolement $\mathrm{n}^{\circ} 2$ a été supérieur à celui des autres choux rouges. La faible taille des graines, compensée par un plus grand nombre de grai- 
nes par silique explique cette production plus abondante en graines en dépit même d'un butinage indirect assez fréquent par les abeilles (tabl. 3) et d'une nouaison significativement plus basse (tabl. 5) que celle qui est obtenue avec les choux rouges des isolements $\mathrm{n}^{\circ} 1$ et $\mathrm{n}^{\circ} 3$.

TABL. 5. - Taux de nouaison, nombre de graines par siliques, poids de 1000 graines et poids de graines pour 1000 fleurs butinables des divers choux fourragers hybrides simples.

TAB. 5. - Prozent Samenansatz, Zahl der Samenkörner je Schote, Gewicht von 1000 Körnern und Gewicht der Körner pro 1000 normaler Blüten verschiedener Einfachhybriden des Blattkohls.

\begin{tabular}{|c|c|c|c|c|c|c|}
\hline & & \multicolumn{4}{|c|}{$\mathrm{N}^{0}$ de l'isolement } & \multirow{2}{*}{$\begin{array}{l}\text { Différences } \\
\text { Unterschied }\end{array}$} \\
\hline & & 1 & 2 & 3 & 4 & \\
\hline \multirow{3}{*}{$\begin{array}{l}\text { Taux de nouaison } \\
\text { Prozent Samenansatz }\end{array}$} & $V \quad \stackrel{m}{s / \sqrt{n}}$ & $\begin{array}{c}79,4 \% \\
(15,81)\end{array}$ & $\begin{array}{c}49,2 \% \\
(5,36)\end{array}$ & $\begin{array}{l}93,2 \% \\
(2,55)\end{array}$ & $\begin{array}{l}88,4 \% \\
(8,67)\end{array}$ & \multirow{3}{*}{$\begin{array}{c}* \\
\text { ppds }=26,8 \\
* * * \\
\text { ppds }=9,7\end{array}$} \\
\hline & $R \quad \stackrel{\bar{m}}{s / \sqrt{n}}$ & $\begin{array}{c}94 \% \\
(4,53)\end{array}$ & $\begin{array}{c}69,6 \% \\
(3,92) \\
\end{array}$ & $\begin{array}{l}92,4 \% \\
(2,07)\end{array}$ & $\begin{array}{r}92,2 \% \\
(2,58) \\
\end{array}$ & \\
\hline & $\begin{array}{l}\text { Différences } \\
\text { Unterschied }\end{array}$ & NS & * & NS & NS & \\
\hline \multirow{3}{*}{$\begin{array}{c}\text { Nombre de graines par silique } \\
\text { Körnerzahl pro Schote }\end{array}$} & $V \quad \frac{\bar{m}}{s / \sqrt{n}}$ & $\begin{array}{l}11,51 \\
(1,64)\end{array}$ & $\begin{array}{l}14,39 \\
(2,63)\end{array}$ & $\begin{array}{l}16,71 \\
(1,41)\end{array}$ & & \multirow{3}{*}{$\begin{array}{c}\text { NS } \\
\text { ** } \\
\text { ppds }=5,5\end{array}$} \\
\hline & $R \quad \frac{\bar{m}}{s / \sqrt{n}}$ & $\begin{array}{c}8,14 \\
(1,05) \\
\end{array}$ & $\begin{array}{c}19,49 \\
(1,81)\end{array}$ & $\begin{array}{l}10,01 \\
(2,63)\end{array}$ & & \\
\hline & $\begin{array}{l}\text { Différences } \\
\text { Unterschied }\end{array}$ & NS & NS & NS & & \\
\hline \multirow{3}{*}{$\begin{array}{l}\text { Poids de } 1000 \text { graines (en cg) } \\
\text { Gewicht von } 1000 \text { Körnern }\end{array}$} & $V \quad \stackrel{\bar{m}}{s / \sqrt{n}}$ & $\begin{array}{l}514,0 \\
(27,3)\end{array}$ & $\begin{array}{l}494,8 \\
(34,9)\end{array}$ & $\begin{array}{l}425 \\
(12,7)\end{array}$ & & \multirow{3}{*}{$\begin{array}{c}\text { NS } \\
\text { * } \\
\text { ppds }=97,7\end{array}$} \\
\hline & $R \quad \stackrel{\bar{m}}{s / \sqrt{n}}$ & $\begin{array}{l}529,4 \\
(23,2)\end{array}$ & $\begin{array}{l}379,8 \\
(27,1)\end{array}$ & $\begin{array}{l}497,6 \\
(48)\end{array}$ & & \\
\hline & $\begin{array}{l}\text { Différences } \\
\text { Unterschied }\end{array}$ & NS & $*$ & NS & & \\
\hline $\begin{array}{l}\text { Poids de graines } \\
\text { pour } 1000 \text { fleurs (en g) }\end{array}$ & $V$ & 47 & 35,5 & 65,8 & & \\
\hline $\begin{array}{l}\text { Gewicht der Körner } \\
\text { von } \quad 1000 \quad \text { Blüten } \quad \text { (in } \quad \text { g) }\end{array}$ & R & 40,5 & 51,5 & 46,1 & & \\
\hline
\end{tabular}

\footnotetext{
NS $=$ Non significatif

* = Significatif à $\mathrm{P}=5 \%$

** = Significatif à $\mathrm{P}=1 \%$

*** $=$ Significatif à $\mathrm{P}=1 \%$

NS $=$ Nicht signifikant

$*$ = Signifikant bei $P=5 \%$

** = Signifikant bei $\mathbf{P}=1 \%$

*** = Signifikant bei $\mathrm{P}=1 \%$
} 
Remarquons aussi que les graines les plus grosses (choux rouges des isolements $\mathrm{n}^{\circ} 1$ et 3 et choux verts de l'isolement $\mathrm{n}^{\circ} 1$ ) (tabl. 5) correspondent à un faible nombre de graines par silique, à une bonne nouaison et à une production de graines satisfaisante. Rappelons que les choux verts de l'isolement $n^{\circ} 1$ ont été cependant assez fréquemment visités indirectement par les abeilles (tabl. 3).

Mais les grosses graines (choux verts de l'isolement $n^{\circ} 2$ ) peuvent aussi correspondre à un nombre de graines par silique un peu plus élevé que précédemment, à une nouaison nettement plus faible et à une production de graines un peu moins importante. Le tableau 3 montre d'ailleurs que le butinage indirect a été très fréquent sur ce chou vert. La mauvaise pollinisation obtenue dans ce cas semble liée au butinage indirect observé mais il n'est toutefois pas possible de l'affirmer.

Enfin les graines de petite taille du chou vert de l'isolement $\mathrm{n}^{0} 3$ correspondent à un nombre de graines par silique plus élevé, à une bonne nouaison et à une production de graines supérieure à celle des choux verts des autres isolements comme c'est le cas d'ailleurs du chou rouge de l'isolement $n^{0} 2$ déjà mentionné.

\section{DISCUSSION}

Ces résultats permettent de faire les remarques suivantes :

- les abeilles domestiques ont été les pollinisateurs les plus abondants sur les fleurs de choux fourragers. Elles ont représenté $86,3 \%$ de la population globale contrairement aux bourdons qui ont été peu nombreux. Ceci correspond d'ailleurs aux estimations généralement données par les auteurs dans d'autres pays, sur d'autres crucifères du même groupe. Citons par exemple : RADCHENKo (1964) qui signale la présence en U.R.S.S. de 88 à $97 \%$ d'abeilles domestiques sur colza (Brassica napus L.), de 77 à $94 \%$ sur radis (Raphanus sativus L.) et Aliev (1971) toujours en U.R.S.S., qui en signale de 82,6 à $97 \%$ sur moutarde. Notons aussi que nous en avions trouvé nousmême en 1966 84,6\% sur choux fourragers dans la région de Rennes.

L'activité de butinage de l'abeille domestique plus importante le matin que l'aprèsmidi sur chou fourrager est en accord avec les observations de PoulsEN (1971) et Aliev (1971) sur colza et sur moutarde.

- le trajet effectué par les butineuses pour visiter les fleurs de chou fourrager sur une aire très restreinte limitée par la taille de nos isolements concordent bien aussi avec les observations de Minderhoud (1931); Singh (1950) et Thies (1953). Ceux-ci considèrent en effet que les butineuses au champ peuvent limiter leur aire de butinage dans une zone très réduite quelquefois inférieure à $100 \mathrm{~m}^{2}$.

FAULKNER (1976) a déjà montré que si l'on utilise les abeilles pour la pollinisation et la création d'hybrides de choux de Bruxelles les plantes parentales doivent être de même hauteur et les fleurs de même couleur. Or, dans nos essais les plantes étaient pré- 
cisément de même taille et les fleurs uniformément jaunes. Une importante différence d'attractivité est tout de même apparue en faveur des fleurs de choux rouges. L'effet couleur des fleurs d'une part et taille des plantes d'autre part étant à rejeter, il resterait donc à connaître l'effet dû à d'éventuelles variations au niveau des sécrétions nectarifères tant quantitatives que qualitatives pour expliquer le butinage préférentiel observé.

- les abeilles domestiques semblent adopter le butinage indirect sur choux fourragers pour prélever le nectar seulement lorsque la morphologie même des éléments qui composent leurs fleurs semble le permettre, c'est-à-dire lorsque les abeilles, en posture latérale, peuvent atteindre plus facilement les nectaires externes plus ou moins accessibles, comme elles le font quelquefois sur les fleurs des arbres fruitiers (TASEI, $1976 \mathrm{~b}$ ).

Le butinage indirect sur fleurs de choux fourragers est un peu différent de celui plus connu des fleurs de certaines légumineuses : fèves, féveroles (TASEI, 1976 a) et trèfle violet (JoubERT et al., 1977), lorsque l'abeille est incitée à prélever le nectar par le trou caractéristique préalablement percé par les bourdons (B. terrestris L.). Mais, comme chez ces dernières ainsi que chez les fleurs d'arbres fruitiers, le butinage indirect des fleurs de choux fourragers ne permet pas la pollinisation.

Cependant l'incidence des taux élevés de butinage indirect sur l'efficacité pollinisatrice observée n'a pas toujours été bien évidente. Il n'a pas toujours été possible par exemple d'établir une corrélation entre ces deux phénomènes. On note seulement quelques concordances, les faibles nouaisons des choux verts et des choux rouges d'un isolement (isolement $\mathrm{n}^{\circ} 2$ ) et les taux de butinage indirects élevés de cet isolement semblent en effet le démontrer. Mais il existe aussi des points de discordance comme dans le cas des choux verts d'un autre isolement (isolement $\mathrm{n}^{\circ} 1$ ) qui ont donné de bonnes nouaisons alors que l'on a noté un important taux de butinage indirect. La coïncidence du butinage indirect et d'une mauvaise pollinisation ne s'est finalement manifestée que dans un seul cas (choux verts de l'isolement $\mathrm{n}^{\circ} 2$ ).

Une bonne pollinisation peut donc se produire en dépit même du butinage indirect et quelle que soit son importance. Ainsi on peut penser que le comportement de butinage " négatif " des abeilles domestiques sur certaines fleurs semble parfois compensé par celui, plus important, du butinage " positif " par d'autres abeilles sur ces mêmes fleurs. En effet, les fleurs visitées latéralement pour le prélèvement du nectar par certaines abeilles domestiques ne semblent pas être forcément toujours les mêmes, les taux de nouaison élevés sont la preuve que ces fleurs ont été aussi butinées, ou même " surbutinées " positivement par d'autres abeilles domestiques pour la collecte de pollen et peutêtre même aussi du nectar, assurant ainsi la pollinisation.

Dans tous les cas il n'est pas possible d'affirmer qu'une mauvaise pollinisation ou une mauvaise nouaison soit le seul fait du butinage indirect. En effet, nous ne savons pas s'il y a eu ou non influence d'une ou de plusieurs autres actions combinées ayant favorisé plus ou moins des phénomènes de coulure. On pourrait invoquer par exemple 
l'action du milieu (température et humidité) sur la coulure physiologique et peut-être aussi celle qui est due aux insectes déprédateurs, comme cela a déjà été constaté sur la luzerne (Medicago sativa L.) par Delaude et TASEI (1972), et aux maladies.

\section{CONCLUSION}

En pollinisation libre et dans des conditions naturelles l'Abeille domestique apparaît comme étant l'espèce qui pollinise le plus et qui donne entière satisfaction. Elle exerce une influence favorable sur la nouaison dans la plupart des isolements. Elle joue un rôle très important dans la pollinisation croisee de divers couples de choux fourragers hybrides simples en isolement pour l'obtention d'hybrides doutles plus productifs lorsque les plantes sont de même taille et que les fleurs sont de même couleur. On peut donc compter sur cette espèce pour hybrider le chou fourrager. Les autres espèces et en particulier les bourdons, qui sont de bons pollinisateurs, ne semblent jouer qu'un rôle secondaire.

Mais, la pollinisation du chou fourrager pose de gros problèmes en raison du butinage indirect effectué par les abeilles domestiques et aussi, pour le type d'hybrides recherché ici, en raison de l'attractivité plus importante des fleurs de choux rouges.

Les concordances notées entre les faibles nouaisons et le butinage indirect ne permettent cependant pas de conclure à une relation positive entre ces deux phénomènes. Ainsi le butinage indirect ne semble pas être seul facteur en cause, mais les autres facteurs susceptibles éventuellement de provoquer des phénomènes de coulure restent à déterminer.

Enfin cette étude, bien qu'incomplète, a permis de formuler des hypothèses qu'il serait intéressant de vérifier : hypothèses concernant les rapports pouvant exister entre le butinage indirect et la morphologie de la fleur, les rapports pouvant exister entre les différents types d'hybrides simples de chou fourrager et le butinage indirect et ceux qui peuvent coexister entre les sécrétions nectarifères et l'att-activité. Il serait aussi interessant de connaitre l'importance relative de divers iacteurs pouvant éventueliement favoriser l'apparition des phénomènes de coulure des divers choux fourragers.

Regit pour fublication en mai 1978.

Eingegungen int Mai 1978 .

\section{ZUSAMMENFASSUNG}

Infolge der Selbst-Inkompatibilität des Pollens ist der Blattkohl mehr oder weniger obligatorisch auf Fremdbestäubung angewiesen. Die Arbeiten zur Verbesserung des Blattkohls bei der I.N.R.A. konzentrieren sich vor allem auf die Erforschung dieses Phänomens, mit dem Ziele der Schaffung von Doppelhybriden. Auf experimenteller Stufe wurde dies durch isolierte Pflanzungen im freien Feld erreicht, wobei man von einigen Paaren von Einfachhybriden ausging. In solchen isolierten Pflanzungen sind unsere Beobachtungen über die Bestäubung durch lokale blütenbesuchende Insekten erfolgt. 
Die Beobachtungen haben sich auf den Ablauf des Blühens, auf die Fauna der Blütenbesucher, auf das Sammelverhalten der Honigbiene (Apis m. mellifica L.) und der Hummeln (Bombus sp.) sowie auf den Effekt der Bestäubung erstreckt.

Es standen vier isolierte Felder zur Verfügung, von denen jedes 20 Pflanzen Einfachhybriden des grünen Blattkohls "Demi-Moellier" $(429 \times 813$ und $429 \times 905)$ und 20 Pflanzen eines anderen Einfachhybriden des roten Blattkohls "Cavalier " $(919 \times 155$ und $111 \times 155)$ enthielt. Die Pflanzen waren in zwei Fällen in alternierenden Reihen, in den beiden anderen Fällen in Form alternierender Pflanzen angeordnet.

Die Ergebnisse zeigten folgendes :

- Die Blüte von Grünkohl und von Rotkohl erfolgt auf allen isolierten Feldern zur selben Zeit.

- Als Bestäuber treten vor allem Honigbienen auf $(86,3 \%)$; ausserdem verschiedene Hummeln $(5,4 \%)$, Wildbienen $(9,2 \%)$ und wenige Dipteren und Lepidopteren $(1.1 \%)$.

- Die Honigbienen hatten manchmal die Tendenz, auf indirektem Wege zu sammeln. Dieses Sammeln wird deshalb als "indirekt" bezeichnet, weil sich die Bienen von aussen an die Basis der Blütenkrone setzen und ausschliesslich Nektar saugen, indem sie die Petale an ihrer Basis leicht auseinanderdrücken. Dieses Verhalten ist zweifellos für die Bestäubung ungünstig. Entsprechend der Häufigkeit des indirekten Sammelns konnte man übrigens Unterschiede zwischen den einzelnen HybridenKombinationen feststellen und danach unterschiedliche Gruppen bilden. Am häufigsten wurden indirekte Besuche beim Grünkohl der Hybridkombination $(429 \times 813)$ beobachtet.

- Es wurde beobachtet, dass die Honigbienen weniger schnell sammeln als die Hummeln. Im Mittel haben sie 5,85 $( \pm 0,56)$ Blüten per Minute gesammelt gegenüber 13,88 $( \pm 2,03)$ Blüten per Minute bei den Hummeln. Die Besuche der Blüten des Rotkohls erfolgten bei beiden Gruppen im allgemeinen rascher als beim Grünkohl. Der Rotkohl hat sich ausserdem als attraktiver gezeigt. In allen Fällen wurde beobachtet, dass der Wechsel des Besuchs zwischen Grünkohl und Rotkohl regelmässig und zufällig erfolgt ist. Beim Überwechseln während des Sammelns hat es weder bei den Bienen noch bei den Hummeln eine gerichtete Orientierung gegeben. Keine der beiden Gruppen zeigte die Tendenz, der Linie oder der Reihe des Blattkohls zu folgen oder sich auf eine bestimmte Linie zu fixieren. Die Honigbiene sammelt den ganzen Tag über, mit einem Maximum am Morgen zwischen 9-11 h, die Hummeln scheinen am Abend ihre Aktivität später zu beenden, gegen $18 \mathrm{~h}$, und das Aktivitätsmaximum liegt am Nachmittag. Die Tagesaktivität der Wildbienen ist kürzer $(9 \mathrm{~h} 30-15 \mathrm{~h}$ ) und am Morgen stärker.

- Die Effektivität der Bestäubung war im ganzen sehr zufriedenstellend. Der Samenansatz war jedoch bei den Kohlptlanzen mit häufigem indirekten Bienenbesuch geringer. Der Rotkohl, attraktiver und von den Bienen tascher besucht, ergab den besseren Samenansatz, in allen Fällen war die Samenproduktion gut.

- Damit ist taachgeviesen, dass die Honigbiene der wichtigste Bestäuber des Kohls ist und dass sie völlig zufriedenstellt. Die Atswirkungen auf den Samenansatz waren günstig. Sie hat eine wichtige Rolle bei der Kreuzbestäubung gespielt, bei Pflanzen, deren Blüten dieselbe Form und dieselbe Farbe zeigen. Die übrigen als Bestäuber tätizen Insekten spielten nur eine sekundäre Rolle.

Infolge des indirekten Sammelns und des Unterschieds in der Attraktivität zwischen Grünkohl und Rotkohl entstehen jedoch gewisst Probleme für die Bestäubung. Aber diese Faktoren scheinen nicht die einzige Ursache für die beobachtete Variabilität beim Samenansatz zu sein. Diese Variabilität scheint von Phänomenen des Blütenfalls unbestimmten Ursprungs abzuhängen, für die sich indessen einige interessante Hypothesen aufstellen lassen. Eine künftige Überprüfung wäre wünschenswert. 


\section{BIBLIOGRAPHIE}

Aliev T. A., 1971. - Utilisation des abeilles mellifères pour la pollinisation de la moutarde. Congr. Intern. Apic., 23, Moscou. C.R., 541-544.

Attia M. S., Munger H. M., 1950. - Self-incompatibility and the production of hybrid cabbage seed. Proc. Amer. Soc. Hort. Sci., 56, 363-368.

Crenu G. du, 1964. - La biologie florale du chou fourrager; ses conséquences pour la sélection. $A n n$. A mél. Plantes, 14, 5-37 et 157-201.

Delaude A., TASEI J. N., 1972. - Premières observations sur la pollinisation et la coulure des fleurs de luzerne en Charentes (Medicago sativa L.). Apidologie, 3, 79-97.

FAULKNER G. J., 1976. - Honeybee behaviour as affected by plant height and flower colour in brussels sprouts. J. of Apic. Res., 15, 15-18.

FreE J. B., 1970. Insect pollination of crops. London. New-York. Academic Press 555 p.

Joubert G., TASEI J. N., Delaude A., 1977. - Pollinisation du trèfle violet diploïde (Trifolium pratense L.) par les Apoïdes et particulièrement par les abeilles domestiques hybrides (Apis mellifica caucasica $\times$ Apis mellifica ligustica). Apidologie, 8, 259-280.

Lecomte J., 1968. - Le comportement de butinage, in Chauvin R. - Traité de biologie de l'A beille, 2, 402-415. Masson, Paris.

Minderhoud A., 1931. - In Lecomte, 1968.

PeArson O. H., 1929. - Observations on the type of sterility in Brassica oleracea var. capitata. Proc. Amer. Soc. Hort. Sci., 26, 34-38.

PoulsEn M. H., 1971. - Mode de travaux et rôle de l'abeille (Apis L.) et des bourdons (Bombus Latr.) dans la production de graines chez la féverole (Vicia faba L.). Thèse Copenhague (en Danois). In TASEI, 1976.

Radchenko T. G., 1964. - In Free, 1970.

Roemer T., 1916. - In du Crehu, 1964.

SiNGH S., 1950. - Behaviour studies of honey-bees in gathering nectar and pollen. Bull. Cornell. Agric. Exp. Sta., $\mathrm{n}^{\circ} 288$.

TASEI J. N., 1976 a. - Les insectes pollinisateurs de la féverole d'hiver (Vicia faba equina L.) et la pollinisation des plantes mâle-stériles en production de semence hybride. Apidologie, 7, 1-38.

TASEI J. N., 1976 b. - Pollinisation des cultures et insectes pollinisateurs. Bul. Techn. Apicole, 3, 15-32.

Thies S. A., 1953. - In Free, 1970. 